\title{
Field-induced phase transitions in relaxor ferroelectrics
}

\section{Authors: V. Hugo Schmidt, R. R. Chien, and C.-S. Tu}

NOTICE: this is the author's version of a work that was accepted for publication in Ferroelectrics. Changes resulting from the publishing process, such as peer review, editing, corrections, structural formatting, and other quality control mechanisms may not be reflected in this document. Changes may have been made to this work since it was submitted for publication. A definitive version was subsequently published in Ferroelectrics, VOL\# 400, ISSUE\# 1, (2010) DOI\# 10.1080/00150193.2010.505879.

V. Hugo Schmidt, R. R. Chien, and C.-S. Tu, "Field-induced phase transitions in relaxor ferroelectrics," Ferroelectrics 400, 402-409 (2010). doi: 10.1080/00150193.2010.505879. 


\title{
Field-Induced Phase Transitions in Relaxor Ferroelectrics
}

\author{
V. HUGO SCHMIDT, ${ }^{1, *}$ R. R. CHIEN,${ }^{1}$ AND CHI-SHUN TU ${ }^{2}$ \\ ${ }^{1}$ Department of Physics, Montana State University, Bozeman, MT 59717, USA \\ ${ }^{2}$ Graduate Institute of Applied Science and Engineering and Department of \\ Physics, Fu Jen Catholic University, Taipei 242, Taiwan, R.O.C
}

\begin{abstract}
The Landau free energy expansion as a function of three-dimensional polarization for perovskite relaxor ferroelectrics is examined to find effects of large electric fields in causing transitions from one ferroelectric state to another. Our examination of fieldinduced effects includes a fourth order expansion to obtain the Landau parameters for the observed temperature-and field-induced rhombohedral $(R) \rightarrow$ tetragonal $(T)$ transitions in an (001)-cut 0.70Pb $\left(\mathrm{Mg}_{1 / 3} \mathrm{Mn}_{2 / 3}\right) \mathrm{O}_{3}-0.30 \mathrm{PbTiO}_{3}(\mathrm{PMN}-30 \% \mathrm{PT})$ crystal. These parameters enabled us to predict the appearance of an orthorhombic $(O)$ phase in (110)-cut PMN-30\%PT crystals under an applied field. These results show that also for field-induced effects, the Landau expansion shows qualitative agreement with experiment for relaxor ferroelectrics.
\end{abstract}

Keywords Relaxor ferroelectric; phase transitions; polarization

\section{Introduction}

Relaxor ferroelectric single crystals are of interest scientifically and technically because their ferroelectric (FE) phase transitions from one crystal structure to another can be easily induced, by changing temperature or applying pressure or an electric field $\mathbf{E}$. In this work, field-induced transitions will be emphasized.

We analyze these transitions by means of the Landau free energy expansion, and compare analytical and experimental results. The free energy expansion includes several approximations. First, the expansion in powers of polarization $\mathbf{P}$ only includes low powers. Second, different phases, and different domains of the same phase, have different unit cell shapes, so at their boundaries there is mechanical strain, whose energy is not taken into account. Third, the whole crystal is assumed to be in a single phase monodomain, so a transition can occur only when the field reaches the point that the free energy of the original phase no longer has even a local minimum. This means that metastable states are allowed, and the processes of domain nucleation and domain wall migration are ignored. Accordingly, calculated hysteresis loops and coercive fields will tend to be larger than observed ones. Fourth, relaxor ferroelectrics have random unlike ion placements that give rise to random fields and strains that tend to smear out transitions, but the Landau expansion does not consider these effects. 
In spite of these approximations, we will see that a number of general observed features of field-induced transitions agree with the free energy expansion predictions.

\section{The Basic Model}

Uniaxial ferroelectrics have Gibbs free energy $\mathrm{G}$ in terms of $\mathrm{P}$ given by a Landau expansion as

$$
\mathrm{G}=-\mathrm{PE}+\mathrm{AP}^{2}+\mathrm{BP}^{4}+\mathrm{CP}^{6}+\cdots
$$

The Curie-Weiss temperature $\mathrm{T}_{0}$ occurs where $\mathrm{A}=\mathrm{A}_{0}\left(\mathrm{~T}-\mathrm{T}_{0}\right)=0$. Symmetry requires only even powers of $\mathrm{P}$, except for the $-\mathrm{PE}$ term.

Perovskite relaxor ferroelectrics that we consider here have a cubic parent paraelectric (PE) phase, so besides these requirements, we must also require that $\mathrm{P}_{\mathrm{x}}^{2}, \mathrm{P}_{\mathrm{y}}^{2}$, and $\mathrm{P}_{\mathrm{z}}^{2}$ enter symmetrically. The subscripts refer to the PE cubic unit cell axes. For simplicity, we denote $\mathrm{P}_{\mathrm{x}}=\mathrm{x}, \mathrm{P}_{\mathrm{y}}=\mathrm{y}$, and $\mathrm{P}_{\mathrm{z}}=\mathrm{z}$. To have a FE transition without runaway $\mathrm{P}$, one must go to 4 th order in $\mathrm{P}$.

Beside the parent cubic (C) PE phase, this 4th order expansion (in zero field) only allows tetragonal (T) and rhombohedral (R) FE phases, as was shown by Devonshire [1]. He found that the 6th order expansion also permits the orthorhombic $(\mathrm{O})$ phase. Vanderbilt and Cohen [2] extended this work and showed that monoclinic $\mathrm{M}_{\mathrm{A}}, \mathrm{M}_{\mathrm{B}}$, and $\mathrm{M}_{\mathrm{C}}$ phases are allowed in the 8th order expansion, and that only in 12th order can the (so far unobserved) triclinic (Tr) FE phase occur.

\section{Fourth Order Expansion Coefficients Found from Experiment}

For analyzing effects of large applied $\mathbf{E}$, the 4th order expansion is the most important. We will show that the $\mathrm{O}$ phase is the stable phase for a certain $\mathbf{E}$ range, even though it cannot exist in zero field in this 4th order expansion. We will also find 4th order expansion coefficients from previously published experimental results.

Expansion terms of higher order than 4th can be considered as "perturbations" on the 4th order terms. Some justification for this statement is that the $\mathrm{O}$ and $\mathrm{M}$ phases in these perovskite relaxor ferroelectrics occur only over narrow ranges in the temperaturecomposition phase diagrams. This is why only the $\mathrm{T}$ and $\mathrm{R}$ phases were known initially.

We write the Gibbs free energy in the 4th order expansion in the most general way that preserves the cubic symmetry:

$$
\mathrm{G}=\mathrm{A}_{2}\left(\mathrm{x}^{2}+\mathrm{y}^{2}+\mathrm{z}^{2}\right)+\mathrm{B}_{4}\left(\mathrm{x}^{4}+\mathrm{y}^{4}+\mathrm{z}^{4}\right)+2 \mathrm{~B}_{22}\left(\mathrm{x}^{2} \mathrm{y}^{2}+\mathrm{y}^{2} \mathrm{z}^{2}+\mathrm{z}^{2} \mathrm{x}^{2}\right)-\mathrm{E}_{\mathrm{x}} \mathrm{x}-\mathrm{E}_{\mathrm{y}} \mathrm{y}-\mathrm{E}_{\mathrm{z}} \mathrm{z}
$$

To have nonzero $\mathbf{P}$ without runaway $\mathbf{P}$, we require $\mathrm{A}_{2}<0$ and $\mathrm{B}_{4}>0$. What requirements apply to $\mathrm{B}_{22}$ ? The $\mathrm{B}_{22}$ term has greatest effect in a body diagonal direction such as [111], for which the three $\mathbf{P}$ components are equal, $\mathrm{x}=\mathrm{y}=\mathrm{z}$, so

$$
\mathrm{G}_{111}=3 \mathrm{~A}_{2} \mathrm{z}^{2}+3 \mathrm{~B}_{4} \mathrm{z}^{4}+6 \mathrm{~B}_{22} \mathrm{z}^{4}
$$

To prevent runaway $\mathrm{P}_{111}$ we must keep $\mathrm{G}_{111}$ positive for large $\mathrm{z}$, which gives us the third requirement, $\mathrm{B}_{22}>-\mathrm{B}_{4} / 2$. This restricts us to the $3 \mathrm{D}$ parameter space

$$
\left(\mathrm{A}_{2}<0, \mathrm{~B}_{4}>0, \mathrm{~B}_{22}>-\mathrm{B}_{4} / 2\right)
$$

for any allowed FE phase. For the cubic PE phase, $T>T_{0}$ in $A_{2}=A_{20}\left(T-T_{0}\right)$. 
Now we investigate which FE phases are allowed in the 4th-order Landau expansion. Given the free energy $\mathrm{G}$ in Eq. (2), an equilibrium (stable, neutral, or unstable) polarization $\mathbf{P}$ exists where the gradient $\nabla G$ vanishes:

$$
\begin{aligned}
\nabla \mathrm{G}= & \hat{i}\left[2 \mathrm{~A}_{2} \mathrm{x}+4 \mathrm{~B}_{4} \mathrm{x}^{3}+4 \mathrm{~B}_{22}\left(\mathrm{y}^{2}+\mathrm{z}^{2}\right) \mathrm{x}-\mathrm{E}_{\mathrm{x}}\right] \\
& +\hat{j}\left[2 \mathrm{~A}_{2} \mathrm{y}+4 \mathrm{~B}_{4} \mathrm{y}^{3}+4 \mathrm{~B}_{22}\left(\mathrm{x}^{2}+\mathrm{z}^{2}\right) \mathrm{y}-\mathrm{E}_{\mathrm{y}}\right] \\
& +\hat{k}\left[2 \mathrm{~A}_{2} \mathrm{z}+4 \mathrm{~B}_{4} \mathrm{z}^{3}+4 \mathrm{~B}_{22}\left(\mathrm{x}^{2}+\mathrm{y}^{2}\right) \mathrm{z}-\mathrm{E}_{\mathrm{z}}\right]=0 .
\end{aligned}
$$

To determine stability, we must take the second gradient and diagonalize the resulting tensor. For stability, all three elements of this diagonalized tensor must be positive. The second gradient is given by the 2 nd rank tensor $\nabla(\nabla G)$ :

$$
\left[\begin{array}{ccc}
2 \mathrm{~A}_{2}+12 \mathrm{~B}_{4} \mathrm{x}^{2}+4 \mathrm{~B}_{22}\left(\mathrm{y}^{2}+\mathrm{z}^{2}\right) & 8 \mathrm{~B}_{22} \mathrm{xy} & 8 \mathrm{~B}_{22} \mathrm{xz} \\
8 \mathrm{~B}_{22} \mathrm{xy} & 2 \mathrm{~A}_{2}+12 \mathrm{~B}_{4} \mathrm{y}^{2}+4 \mathrm{~B}_{22}\left(\mathrm{x}^{2}+\mathrm{z}^{2}\right) & 8 \mathrm{~B}_{22} \mathrm{yz} \\
8 \mathrm{~B}_{22} \mathrm{xz} & 8 \mathrm{~B}_{22} \mathrm{yz} & 2 \mathrm{~A}_{2}+12 \mathrm{~B}_{4} \mathrm{z}^{2}+4 \mathrm{~B}_{22}\left(\mathrm{x}^{2}+\mathrm{y}^{2}\right)
\end{array}\right]
$$

We begin by considering two cases for which $\nabla(\nabla G)$ is already diagonal. The first is for $\mathbf{E}=\hat{k} \mathrm{E}$, which favors one of the six T domains. For that domain, $\mathrm{x}=\mathrm{y}=0$ and $\mathrm{z}>0$ in Eqs. (5) and (6). The solution for Eq. (5) then is

$$
\nabla \mathrm{G}=\hat{k}\left[2 \mathrm{~A}_{2} \mathrm{z}+4 \mathrm{~B}_{22} \mathrm{z}^{3}-\mathrm{E}\right]=0
$$

For $\mathrm{E}=0$ in Eq. (7), $\mathrm{z}^{2}=-\mathrm{A}_{2} / 2 \mathrm{~B}_{4}$. Inserting this value for $\mathrm{z}^{2}$ and 0 for $\mathrm{x}$ and $\mathrm{y}$ into Eq. (6) yields the following "curvatures" of $\mathrm{G}$ in the $\mathrm{x}, \mathrm{y}$, and $\mathrm{z}$ directions respectively, using the notation $\partial^{2} \mathrm{G} / \partial \mathrm{x}^{2} \equiv \mathrm{G}_{\mathrm{xx}}$, etc.:

$$
G_{x x}=G_{y y}=2 A_{2}-2 A_{2} B_{22} / B_{4}, G_{z z}=-4 A_{2} .
$$

From Eq. (8) we see that for zero field the stability requirement for $\mathrm{T}$ domains is $\mathrm{B}_{22}>\mathrm{B}_{4}$. This means that $\mathrm{T}$ domains are stable in only that part of one parameter space octant for which $\left(\mathrm{A}_{2}<0,0<\mathrm{B}_{4}<\mathrm{B}_{22}\right)$.

The other case for which $\nabla(\nabla G)$ is already diagonal is for $B_{22}=0$. In this case, Eq. (5) becomes

$$
\nabla \mathrm{G}=\hat{i}\left[2 \mathrm{~A}_{2} \mathrm{x}+4 \mathrm{~B}_{4} \mathrm{x}^{3}-\mathrm{E}_{\mathrm{x}}\right]+\hat{j}\left[2 \mathrm{~A}_{2} \mathrm{y}+4 \mathrm{~B}_{4} \mathrm{y}^{3}-\mathrm{E}_{\mathrm{y}}\right]+\hat{k}\left[2 \mathrm{~A}_{2} \mathrm{z}+4 \mathrm{~B}_{4} \mathrm{z}^{3}-\mathrm{E}_{\mathrm{z}}\right]=0,
$$

and Eq. (6) yields the following expression for $\nabla(\nabla G)$ :

$$
\left[\begin{array}{ccc}
2 \mathrm{~A}_{2}+12 \mathrm{~B}_{4} \mathrm{x}^{2} & 0 & 0 \\
0 & 2 \mathrm{~A}_{2}+12 \mathrm{~B}_{4} \mathrm{y}^{2} & 0 \\
0 & 0 & 2 \mathrm{~A}_{2}+12 \mathrm{~B}_{4} \mathrm{z}^{2}
\end{array}\right]
$$

For $\mathbf{E}=0$, Eq. (9) shows that $\mathrm{x}^{2}, \mathrm{y}^{2}$, and $\mathrm{z}^{2}$ separately can have values 0 or $-\mathrm{A}_{2} / 2 \mathrm{~B}_{4}$. Only if they are all nonzero is there a stable phase, the $\mathrm{R}$ phase.

Devonshire [1] showed that in the 4th-order Landau expansion the only stable phases are $\mathrm{T}$ or $\mathrm{R}$ for $\mathbf{E}=0$. For $\mathbf{E}$ applied along [110], we find from Eqs. (5) and (6) that an $\mathrm{O}$ phase with $\mathbf{P}$ along [110] is the stable phase in a certain field range, as shown in Fig. 1. In 4th order there are no metastable phases, and field- and temperature-induced transitions are of 2 nd order. 


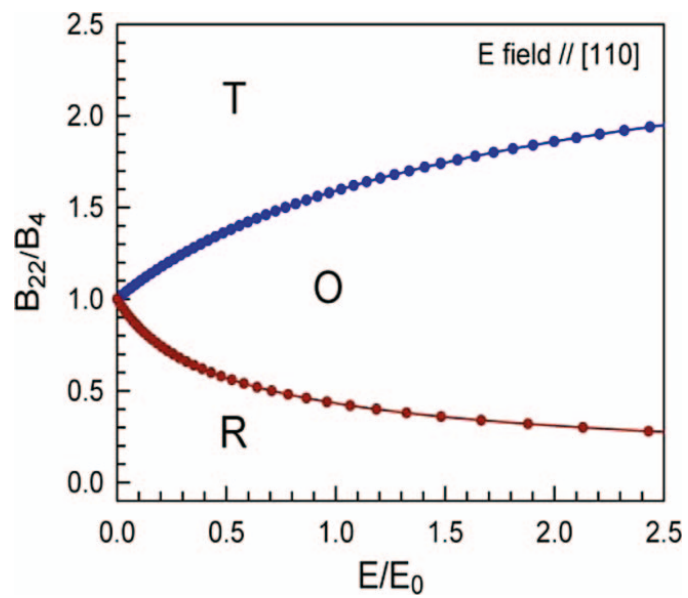

Figure 1. Field-induced orthorhombic phase in 4th order Landau expansion for E field along [110]. The "reference field" $\mathrm{E}_{0}$ is defined as $\mathrm{E}_{0} \equiv\left[2\left(-\mathrm{A}_{2}\right)^{3} / \mathrm{B}_{4}\right]^{1 / 2}$. (See Color Plate LXII)

The next step is determination of Landau expansion coefficients from experimental results. We start with

$$
\mathrm{A}_{2}=\mathrm{A}_{20}\left(\mathrm{~T}-\mathrm{T}_{0}\right)
$$

We find $\mathrm{T}_{0}$ by plotting inverse permittivity $\varepsilon^{-1} v s$. temperature $\mathrm{T}$ in the PE phase where $\mathrm{T}>\mathrm{T}_{0}$, noting that in cubic crystals $\varepsilon$ is isotropic. Then $\mathrm{T}_{0}$ is the temperature where the extrapolation of the straight line portion of this plot crosses the $\mathrm{T}$ axis. For relaxor ferroelectrics this is a rather long extrapolation that begins at the Burns temperature $T_{B}$ below which polar clusters form. These clusters reduce $\varepsilon$ below the value predicted by the Curie-Weiss law

$$
\varepsilon=\mathrm{C} /\left(\mathrm{T}-\mathrm{T}_{0}\right)
$$

where $\mathrm{C}$ is the Curie-Weiss constant. To relate $\mathrm{C}$ to Landau expansion coefficients, we first use the relation

$$
\mathrm{D}=\varepsilon_{0} \mathrm{E}+\mathrm{P}=\varepsilon_{0} \varepsilon \mathrm{E} .
$$

For most FE materials in the PE phase, the vacuum term $\varepsilon_{0} \mathrm{E}$ is much less than $\mathrm{P}$ and can be neglected. Then, Eqs. (11-13) give

$$
\varepsilon=\mathrm{P} / \varepsilon_{0} \mathrm{E}=\mathrm{C} /\left(\mathrm{T}-\mathrm{T}_{0}\right)=\mathrm{CA}_{20} / \mathrm{A}_{2} .
$$

In the PE phase, $\mathrm{A}_{2}>0$ and for permittivity measurements $\mathrm{E}$ is small, so only the linear terms in Eq. (5) are important. This gives, for any $\mathbf{E}$ direction,

$$
\mathrm{P}=\mathrm{E} / 2 \mathrm{~A}_{2} \text {. }
$$

Inserting this P value into Eq. (14) and using Eq. (11) yields

$$
\mathrm{A}_{20}=1 / 2 \varepsilon_{0} \mathrm{C} \text {. }
$$


Now that $A_{20}$ is found from experimental data, we can find $B_{4}$ from the measured saturation spontaneous polarization if the FE phase is tetragonal. From Eq. (7) we found that polarization $\mathrm{P}_{\mathrm{T}}$ for the $\mathrm{T}$ phase is

$$
\mathrm{P}_{\mathrm{T}}=\left(-\mathrm{A}_{2} / 2 \mathrm{~B}_{4}\right)^{1 / 2} \text {. }
$$

Inserting the $\mathrm{A}_{2}$ expression from Eqs. (11) and (16) into Eq. (17) gives

$$
\mathrm{B}_{4}=\left(\mathrm{T}_{0}-\mathrm{T}\right) / 4 \varepsilon_{0} \mathrm{CP}_{T}^{2} \text {. }
$$

Solving for $\mathrm{P}_{\mathrm{T}}$ yields

$$
\mathrm{P}_{\mathrm{T}}=\left[\left(\mathrm{T}_{0}-\mathrm{T}\right) / 4 \varepsilon_{0} \mathrm{CB}_{4}\right]^{1 / 2} .
$$

This means that if $\mathrm{B}_{4}$ has negligible $\mathrm{T}$ dependence, the spontaneous polarization is predicted to vary as the square root of $\left(\mathrm{T}_{0}-\mathrm{T}\right)$. This dependence will change as higher order terms are introduced into the Landau expansion.

To find the remaining 4 th order Landau expansion, $B_{22}$, we note that $B_{22}=B_{4}$ at the $\mathrm{R} \rightarrow \mathrm{T}$ transition temperature.

We now find numerical values for $\mathrm{A}_{20}, \mathrm{~B}_{4}$, and $\mathrm{B}_{22}$ for a $<001>-$ cut PMN-30\%PT single crystal, by inserting experiment results [3] into the above equations. The Curie-Weiss constant $\mathrm{C}$ from permittivity data above the Burns temperature of $510 \mathrm{~K}$ is $1.8 \times 10^{5} \mathrm{~K}$, and the Curie-Weiss temperature $\mathrm{T}_{0}$ is $465 \mathrm{~K}$. As typical for relaxor ferroelectrics, the transition out of the PE phase occurred well below $\mathrm{T}_{0}$, at $410 \mathrm{~K}$.

Using these parameters in Eq. (16), we find $\mathrm{A}_{20}$ to be given by

$$
\mathrm{A}_{20}=3.8 \times 10^{5} \mathrm{~J}-\mathrm{m} / \mathrm{C}^{2} \mathrm{~K} .
$$

To find $\mathrm{B}_{4}$ from Eq. (17), we first need a value for $\mathrm{P}_{\mathrm{T}}$ at some temperature. For $\mathrm{B}_{4}$ to be independent of temperature, $\mathrm{P}_{T}^{2}$ must vary as $\mathrm{T}_{0}-\mathrm{T}$. From hysteresis loop plots in Fig. 2a of Ref. [3], this temperature dependence is satisfied between 370 and $420 \mathrm{~K}$, with

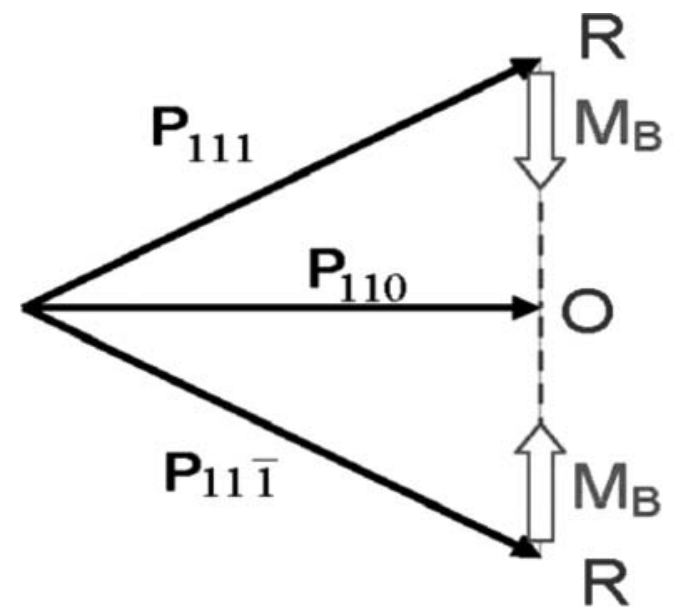

Figure 2. Schematic of [110] E-field-induced polarization rotation into $\mathbf{M}_{B}$ directions (arrows) followed by 1st-order polarization jump (dashed lines), in the process that converts rhombohedral phase to orthorhombic phase. 
$\mathrm{P}_{\mathrm{s}}=0.158 \mathrm{C} / \mathrm{m}^{2}$ at $400 \mathrm{~K}$. Using this $\mathrm{P}_{\mathrm{s}}, \mathrm{B}_{4}$ is given by

$$
\mathrm{B}_{4}=4.09 \times 10^{8} \mathrm{~J}-\mathrm{m}^{5} / \mathrm{C}^{4} .
$$

From polarizing microscopy pictures in Fig. 10 of Ref. [3], at $300 \mathrm{~K}$ the field-induced $\mathrm{R} \rightarrow \mathrm{T}$ transition occurs at $2 \times 10^{6} \mathrm{~V} / \mathrm{m}$. Then from the Landau expansion we find that $\mathrm{B}_{22} / \mathrm{B}_{4}=0.95$ at $300 \mathrm{~K}$. At zero field the $\mathrm{R} \rightarrow \mathrm{T}$ transition occurs at $355 \mathrm{~K}$ based on permittivity and polarization current density plots in Fig. 1 of Ref. [3], and on X-ray diffraction data in Fig. 9 of Ref. [3]. Thus the Landau expansion requires that $\mathrm{B}_{22}=\mathrm{B}_{4}$ at $355 \mathrm{~K}$. We do not know which of $\mathrm{B}_{4}$ or $\mathrm{B}_{22}$ has the stronger temperature dependence. If for simplicity we assume $\mathrm{B}_{4}$ is independent of temperature, then from the above numbers we obtain for an assumed linear dependence of $\mathrm{B}_{22}$ on $\mathrm{T}$ the expression

$$
\mathrm{B}_{22}=\left[1+9.09 \times 10^{-4} \mathrm{~K}^{-1}(\mathrm{~T}-355 \mathrm{~K})\right] \mathrm{B}_{4} .
$$

\section{Landau Expansion Fit to Field-Induced Rhombohedral to Orthorhombic Transition}

We now discuss the appearance of the orthorhombic O phase in PMN-PT crystals as a function of Ti concentration $\mathrm{x}$, temperature, field, and crystal cut, based on some experimental observations [4-7]. For $\mathrm{x}=0.33$, some but not all (011)-cut crystals showed an $\mathrm{O}$ monodomain phase after poling with $\mathrm{E}>5 \times 10^{5} \mathrm{~V} / \mathrm{m},{ }^{4}$ but only an R polydomain state after poling with $\mathrm{E}<4 \times 10^{5} \mathrm{~V} / \mathrm{m}$. For an $\mathrm{x}=0.30$ (011)-cut crystal at room temperature, application of unipolar field showed an anhysteretic $\mathrm{P}(\mathrm{E})$. With increasing $\mathrm{E}, \mathbf{P}$ rotation along monoclinic $\mathrm{M}_{\mathrm{B}}$ directions first occurred, followed by a first-order transition to the $\mathrm{O}$ phase [5], as illustrated in Fig. 2. In a comparison of (001)-, (011)-, and (111)-cut crystals having $\mathrm{x}=0.24,0.30,0.33$, and 0.38 [6], after poling at $10^{6} \mathrm{~V} / \mathrm{m}$ at the dielectric maximum and cooling to room temperature, the permittivity curves upon heating were interpreted to show no $\mathrm{O}$ phase for $\mathrm{x}=0.24$ and $\mathrm{O}$ phases only for the (011)-cut crystals for the other samples. For the $\mathrm{x}=0.30$ crystal, the $\mathrm{R} \rightarrow \mathrm{O}$ and $\mathrm{O} \rightarrow \mathrm{T}$ transitions took place near $362 \mathrm{~K}$ and $373 \mathrm{~K}$ respectively. In the $\mathrm{x}=0.33$ and $\mathrm{x}=0.38$ (011)-cut crystals, the $\mathrm{O}$ phase was seen from room temperature up to $348 \mathrm{~K}$ and $311 \mathrm{~K}$, respectively. In a (011)-cut PMN-30\%PT crystal [7], an O phase was seen between 355 and $370 \mathrm{~K}$ upon heating after poling at $5 \times 10^{5} \mathrm{~V} / \mathrm{m}$ at room temperature. Without poling, only the $\mathrm{R}$ and $\mathrm{T}$ phases were seen, with the $\mathrm{R} \rightarrow \mathrm{T}$ transition at $378 \mathrm{~K}$.

These results show that the range of the $\mathrm{O}$ phase increases with $\mathrm{x}$ for prior-poled (011)-cut crystals, but the fact that it is only observed in this cut implies that strain effects play an important role, so it is not clear experimentally whether the $\mathrm{O}$ phase is the lowest free energy monodomain phase in any of these crystals.

To predict theoretically whether $O$ phase has the lowest free energy, we must go to the 6th order terms in the Landau expansion. The $\mathrm{O}$ phase is most likely to be found in these PMN-PT crystals for those $\mathrm{x}$ and temperature combinations that give nearly equal free energy for the $\mathrm{R}$ and $\mathrm{T}$ phases. When these 6th order terms are included, the free energy is given by

$$
\begin{aligned}
G & =A_{2}\left(x^{2}+y^{2}+z^{2}\right)+B_{4}\left(x^{4}+y^{4}+z^{4}\right)+2 B_{22}\left(x^{2} y^{2}+y^{2} z^{2}+z^{2} x^{2}\right)+C_{6}\left(x^{6}+y^{6}+z^{6}\right) \\
& +3 C_{42}\left[x^{4}\left(y^{2}+z^{2}\right)+y^{4}\left(z^{2}+x^{2}\right)+z^{4}\left(x^{2}+y^{2}\right)\right]+6 C_{222} x^{2} y^{2} z^{2}-E_{x} x-E_{y} y-E_{z} z .
\end{aligned}
$$




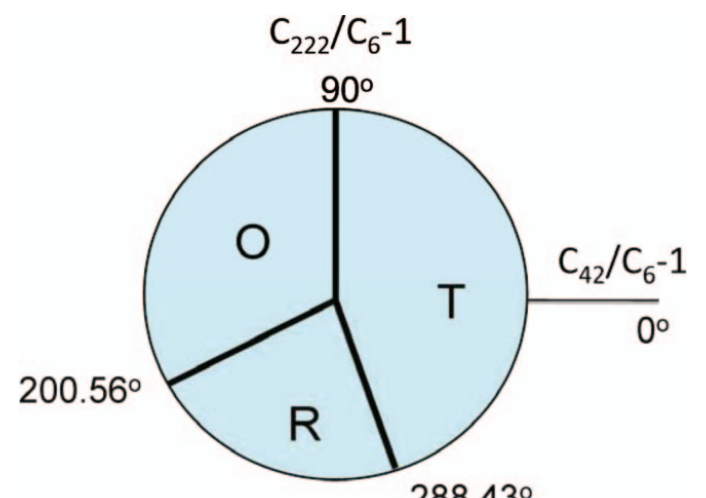

$288.43^{\circ}$

Figure 3. Pie chart showing dependence of phases on 6th order Landau coefficients for the case that the 4 th order coefficients put the crystal at the morphotropic phase boundary.

The numerical factors 2,3 , and 6 above are chosen so that if $\mathrm{B}_{22}=\mathrm{B}_{4}, \mathrm{C}_{222}=\mathrm{C}_{42}=$ $\mathrm{C}_{6}$, and $\mathrm{E}=0$, the free energy is the same for all $\mathbf{P}$ directions.

We can draw a pie chart with horizontal $\left(0^{\circ}\right)$ axis $\mathrm{C}_{42} / \mathrm{C}_{6}-1$ and vertical $\left(90^{\circ}\right)$ axis $\mathrm{C}_{222} / \mathrm{C}_{6}-1$, as shown in Fig. 3. If we retain the conditions $\mathrm{B}_{22}=\mathrm{B}_{4}$ and $\mathrm{E}=0$ that in 4 th order make all $\mathbf{P}$ directions equally likely, then the $\mathrm{O}$ phase will be stable in the wedge between $90^{\circ}$ and $200.56^{\circ}$ [ $\tan ^{-1}(3 / 8)$ ], the $\mathrm{R}$ phase will be stable between $200.56^{\circ}$ and $288.43^{\circ}\left[\tan ^{-1}(-3)\right]$, and the $\mathrm{T}$ phase will be stable between $288.43^{\circ}$ and $90^{\circ}$.

In future work, we hope to gain valuable information on the Landau 6th order expansion coefficients from results such as those of Viehland and Li on (011)-cut PMN-30\%PT at room temperature [5]. For now, we compare their results with predictions based on the 4th order coefficients found in Section 3. Their measurements of $\Delta \mathrm{P}$ as a function of unipolar fields ranging from zero to three different magnitudes, $10^{6}, 2 \times 10^{6}$, and $4 \times 10^{6} \mathrm{~V} / \mathrm{m}$, all fall on the same $\Delta \mathrm{P} v s$. E curve with no hysteresis, which implies that the sample was monodomain and so exhibited no strain effects. (The electrodes were gold and so were unlikely to cause much strain.) The data fall on three straight-line segments with only slight rounding where these segments meet, as shown in Fig. 4. The segment meeting points are at $\left(7.8 \times 10^{5} \mathrm{~V} / \mathrm{m}, 0.033 \mathrm{C} / \mathrm{m}^{2}\right)$ and $\left(10.3 \times 10^{5} \mathrm{~V} / \mathrm{m}, 0.068 \mathrm{C} / \mathrm{m}^{2}\right)$. If the low-field

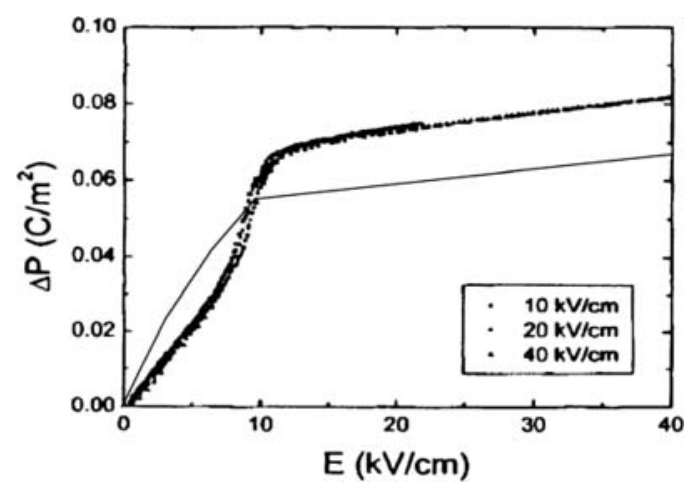

Figure 4. Unipolar polarization vs. field for (110)-cut PMN-30\%PT crystal as found by Viehland and $\mathrm{Li}$ [5], with fine line showing 4th order Landau expansion prediction based on experiments [3] on a (001)-cut PMN-30\%PT crystal. 
segment is extrapolated to the high-field segment, their meeting point is at $\left(16.9 \times 10^{5}\right.$ $\left.\mathrm{V} / \mathrm{m}, 0.072 \mathrm{C} / \mathrm{m}^{2}\right)$. The slopes of the three segments are $23.6 \times 10^{6}, 71.4 \times 10^{6}$, and $8.2 \times$ $10^{6}\left(\mathrm{C} / \mathrm{m}^{2}\right) /(\mathrm{V} / \mathrm{m})$.

The interpretation by Viehland and $\mathrm{Li}$ [5] for this behavior is that the first segment shows polarization rotation from $\mathrm{R}$ toward $\mathrm{O}$ via monoclinic $\mathrm{M}_{\mathrm{B}}$. Then the steep slope between $7.8 \times 10^{5}$ and $10.3 \times 10^{5} \mathrm{~V} / \mathrm{m}$ corresponds to a transition at a critical point where the crystal transforms to the $\mathrm{O}$ phase. We note that there is a slight hysteresis in this steepest segment, which supports the possibility that if domain nucleation and domain wall migration were absent, there would be a polarization jump corresponding to their assumed first-order phase transition.

Now we used the $A_{20}, B_{4}$ and $B_{22}$ values we found above from the 4 th order Landau expansion applied to the temperature- and field-induced $\mathrm{R} \rightarrow \mathrm{T}$ transitions of (001)-cut PMN$30 \% \mathrm{PT}$ to the above-described field-induced $\mathrm{R} \rightarrow \mathrm{O}$ transition for (110)-cut PMN-30\%PT, without introducing any other parameters. This 4th order Landau expansion analysis predicts polarization growth and rotation with increasing field, as illustrated schematically in Fig. 2. The polarization starts at zero field from a [111] or [111] R direction, then changes direction along an $\mathrm{M}_{\mathrm{B}}$-direction path until that path reaches the [110] O-phase polarization direction, with no jump in magnitude or direction at that point. After that, the polarization increases more slowly with field as shown by the fine line in Fig. 4, and remains along [110].

This 4th order expansion does not predict the steepest slope portion seen experimentally, because the 4th order Landau expansion cannot predict a 1 st order transition. It remains to be determined whether 6th order terms would predict a polarization jump. If so, we can try to find the three 6 th order parameters $\mathrm{B}_{6}, \mathrm{~B}_{42}$, and $\mathrm{B}_{222}$ from three experiment parameters (see Fig. 4), namely the $\mathrm{E}$ value and the top and bottom $\Delta \mathrm{P}$ values at the polarization jump.

\section{Conclusion}

The Landau free energy expansion, even if carried out only to 4th order, is shown to be able to predict a field-induced transition in fair agreement with experiment. Experiments in which unipolar fields produce transitions from one monodomain phase to another [5] are particularly helpful in finding Landau expansion coefficients.

\section{References}

1. A.F. Devonshire, Philos. Mag. 40, 1040 (1949).

2. D. Vanderbilt and M. H. Cohen, Phys. Rev. B 63, 094108 (2001).

3. C.-S. Tu, C.-M. Hsieh, R. R. Chien, V. H. Schmidt, F.-T. Wang, and W. S. Chang, J. Appl. Phys. 103, 074117 (2008).

4. Y. Lu, D.-Y. Jeong, Z.-Y. Cheng, Z. M. Zhang, H. Luo, Z. Yin, and D. Viehland, Appl. Phys. Lett. 78, 3109 (2001).

5. D. Viehland and J. F. Li, J. Appl. Phys. 92, 7690 (2002).

6. Z. Feng, X. Zhao, and H. Luo, J. Appl. Phys. 100, 024104 (2006).

7. C.-S. Tu, V. H. Schmidt, R. R. Chien, S.-H. Tsai, S.-C. Lee, and H. Luo, J. Appl. Phys. 104, 094105 (2008). 